Ann. Biol. anim. Bioch. Biophys., 1979, 19 (3 B), 703-707.

\title{
Influence des glucides alimentaires sur la croissance et la vitesse d'absorption intestinale du glucose et du fructose chez le poulet
}

Anne GAUTHIER, M. LARBIER, J. C. BLUM

Station de Recherches Avicoles, I.N.R.A. Nouzilly 37380 Monnaie.

Summary. Effects of dietary carbohydrates on growth and intestinal absorption of glucose and fructose in the growing chick.

Male meat-type chicks were offered three different diets from hatching to 8 weeks of age : two were high in carbohydrate (61 p. 100 sucrose; 61 p. 100 starch), the third was carbohydrate-free ( 26 p. 100 maize oil ; 35 p. 100 cellulose). The growth rate of chickens was greater when high carbohydrate diets, and especially the sucrose-base diet, were fed. Five-week old weights were, respectively, 1087, 910 and $806 \mathrm{~g}$ for sucrose, starch and fat diets. However, feed conversion, between 1 and 5 weeks was lower for sucrose and carbohydrate free diets (1.52 and 1.59) than for starch diets (1.71). Whichever method of analysis or feeding was used, it was confirmed that glucose absorption rate was higher than that of fructose. In vitro accumulation of the two monosaccharides was more marked in the jejunum and ileum than in the duodenum. In vitro and in vivo, the absorption rates were improved by the carbohydrate-free diet. There was no significant difference between starch or sucrose diets.

\section{Introduction.}

Dans les régimes habituellement consommés par les volailles, les glucides constituent la source la plus importante de nutriments énergétiques. De nombreuses études ont été consacrées à la digestion des glucides chez les mammifères alors que d'une bibliographie récente (Levin, 1976), il ressort que les recherches effectuées chez les oiseaux sont plus rares. En particulier, l'influence du régime alimentaire sur l'activité fonctionnelle de l'intestin grêle a été peu envisagée.

Dans cet essai, nous avons recherché dans quelle mesure la consommation de régimes alimentaires privés ou non de glucides (amidon ou saccharose) affecte les performances de croissance du poulet et modifie la vitesse d'absorption de deux hexoses : le glucose et le fructose. 


\section{Matériel et méthodes.}

Des poussins mâles de type chair Hubbard sont élevés en cages individuelles sous un rythme lumineux comportant $16 \mathrm{~h}$ de lumière et $8 \mathrm{~h}$ d'obscurité par nycthémère. Dès la naissance, ils sont répartis en 3 lots de 32 animaux et reçoivent, jusqu'à l'âge de 8 semaines, des régimes complets et équilibrés, semblables par leurs caractéristiques nutritionnelles (isoénergétiques, isoprotidiques, etc...) mais différents par leur apport glucidique : 61 p. 100 de saccharose ; 61 p. 100 d'amidon ; ou absence de glucides digestibles (26 p. 100 de graisse +35 p. 100 de cellulose). La composition exacte de ces régimes est présentée dans le tableau 1.

\section{TABLEAU I}

Composition centésimale des régimes alimentaires

\begin{tabular}{llc}
\hline Régime saccharose & Régime amidon & Régime « sans glucide » \\
\hline Saccharose : 61 & Amidon de maïs : 61 & Huile de maïs : 26 \\
& & Cellulose : 35 \\
\hline
\end{tabular}

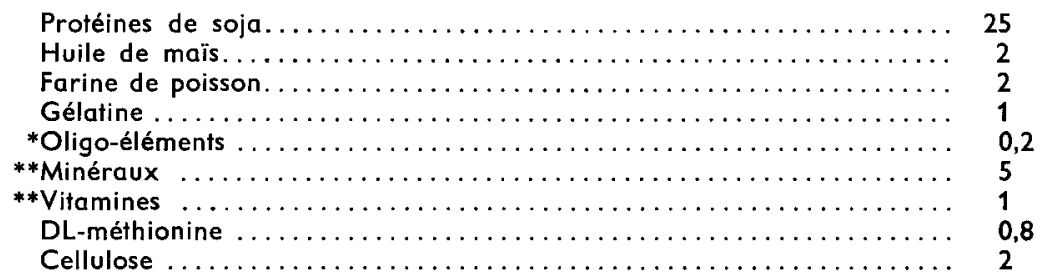

*Oligoéléments (mélange en ppm) : Co:875; Cu $8750 ; 1: 1235 ;$ Se :150; Zn :100000; $\mathrm{Fe}: 35000 ; \mathrm{Mn}: 110000$.

**Vitamines et minéraux (pour $100 \mathrm{~kg}$ d'aliments) : Vit. $A: 1000000 \mathrm{UI}$; Vit. $D_{3}: 150000 \mathrm{UI}$; Vit. $E: 5 \mathrm{~g} ;$ Vit. $\mathrm{K}_{3}: 0,4 \mathrm{~g}:$ Vit. $\mathrm{B}_{1}: 1 \mathrm{~g} ;$ biotine $: 0,04 \mathrm{~g}$; panthoténate de $\mathrm{Ca}: 4 \mathrm{~g} ;$ Vit. $\mathrm{B}_{12}: 0,005 \mathrm{~g}$; Vit. PP: $8 \mathrm{~g}$; Vit. $\mathrm{B}_{6}: 2 \mathrm{~g}$; Vit. $\mathrm{B}_{2}: 1 \mathrm{~g}$; acide folique :0,3 $\mathrm{g}$; chlorure de choline pur : $200 \mathrm{~g}$; B. H. T.: $15 \mathrm{~g}$;

$\mathrm{NaCl}: 0,5 \mathrm{~kg} ; \mathrm{KH}_{2} \mathrm{PO}_{4}: 1,5 \mathrm{~kg} ; \mathrm{CaHPO}_{4}: 0,8 \mathrm{~kg} ; \mathrm{CaCO}_{3}: 1,7 \mathrm{~kg} ; \mathrm{MgSO}_{4}, \mathrm{H}_{2} \mathrm{O}: 0,5 \mathrm{~kg}$.

Caractéristiques (valeurs calculées) :

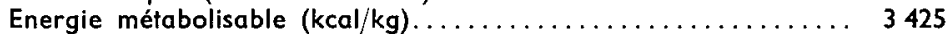

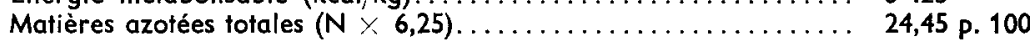

Lysine . . . . . . . . . . .

Acides aminés soufrés. . . . . . . . . . . . . . .

Calcium .............. 1,21 -

Phosphore disponible $\ldots \ldots \ldots \ldots \ldots \ldots \ldots \ldots \ldots \ldots \ldots \ldots \ldots \ldots, 0,57 \quad$ -

Les poids des animaux et la quantité d'aliment consommée sont mesurés une fois par semaine.

La vitesse d'absorption du glucose et du fructose est déterminée in vitro et in vivo. Dans la technique in vitro, les animaux âgés de 4 semaines sont sacrifiés par décapitation après $14 \mathrm{~h}$ de jeûne. Sur chacune des trois anses de l'intestin grêle (duodénum, num et iléon) on prélève des fragments d'intestin de $1,5 \mathrm{~cm}$ de long. Après lavage 
avec du liquide de Tyrode, les fragments sont incubés pendant vingt minutes en milieu Tyrode oxygéné ( $95 \mathrm{p} .100 \mathrm{CO}_{2}$ ef 5 p. $100 \mathrm{O}_{2}$ ) contenant du glucose ${ }^{14} \mathrm{C}(\mathrm{U})$ ou du fruclose ${ }^{14} \mathrm{C}(\mathrm{U})(5 \mathrm{~g} / 1$; activité spécifique : $0,36 \mu \mathrm{Ci} / \mathrm{mM})$. Les fragments sont lavés, séchés et congelés. Les oses accumulés dans les fragments d'intestin sont extraits à l'acide trichloroacétique (à 10 p. 100). La radioactivité est mesurée à l'aide d'un compteur à scintillation liquide.

Dans la méthode in vivo, les poulets à jeun depuis $14 \mathrm{~h}$ sont anesthésiés au pentobarbital sodique. Le duodénum isolé est canulé aux deux extrémités (anse de ThiryVella). L'anse ainsi préparée est lavée avec la solution de Tyrode à $39^{\circ} \mathrm{C}$, puis perfusée en «circuit ouvert» (débit : $0,42 \mathrm{ml} / \mathrm{min}$ ), pendant une heure, avec une solution de Tyrode additionnée de glucose ef de fructose $(5 \mathrm{~g}$ de chaque glucide par litre). Les perfusats sont recueillis dans des éprouvettes graduées de manière à déterminer le volume.

Le glucose est dosé par une méthode enzymatique (glucose-oxydase) à l'autoanalyseur technicon tandis que le fructose est dosé par la méthode de von Handel à l'anthrone froid.

\section{Résultats et discussion.}

1. Performances de croissance (łabl. 2). — Les régimes semi-synthétiques distribués dès la naissance assurent des performances comparables à celles obtenues avec les mélanges alimentaires traditionnels (maïs-soja). On peut donc considérer que les besoins nutritionnels du poulet sont satisfaits et que les variations observées d'un lot à l'autre tiennent seulement à la nature de l'apport énergétique.

TABLEAU 2

Influence du régime alimentaire sur les performances de croissance du poulet

\begin{tabular}{|c|c|c|c|}
\hline \multirow{2}{*}{ Performance } & \multicolumn{3}{|c|}{ Régime } \\
\hline & Saccharose & Amidon & Graisse-cellulose \\
\hline Poids vif à 5 semaines. . . . . . . . & $1087(A)$ & $910(B)$ & $806(C)$ \\
\hline $\begin{array}{l}\text { Indice de consommation de } 1 \text { à } \\
5 \text { semaines }(\mathrm{kg} \text { d'aliment } / \mathrm{kg} \text { de } \\
\text { gain de poids).............. }\end{array}$ & $1,52 \pm 0,037\left({ }^{a}\right)$ & $1,71 \pm 0,015(b)$ & $1,59 \pm 0,029\left({ }^{c}\right)$ \\
\hline
\end{tabular}

Les valeurs suivies de lettres distinctes sont significativement différentes : lettres majuscules $(P<0,01)$ ou minuscules $(P<0,05)$.

Les meilleurs résultats sont obtenus avec les régimes riches en glucides. Comparé à l'amidon, le saccharose permet des gains de poids plus importants. Les indices de consommation déterminés entre 1 et 5 semaines d'âge sont égaux à 1,52 pour le lot saccharose ef 1,71 pour le lot amidon. Cette supériorité du saccharose n'a pas souvent été mise en évidence. En effet, la plupart des recherches reconnaissent des qualités égales au saccharose et à l'amidon pendant la croissance du poussin (Siddons, 1972). 
Dans le cas du régime privé de glucides (le dosage des matières premières indique une teneur en glucides solubles inférieurs à 0,05 p. 100) les performances de croissance sont notablement réduites. A cinq semaines, le poids vif est inférieur de 25 p. 100 à celui obtenu avec l'aliment à base de saccharose. Dans le même temps, l'indice de consommation augmente légèrement $\left(I_{c}=1,59\right)$. Nous confirmons ainsi plusieurs observations tendant à montrer que les régimes riches en lipides et cellulose n'assurent pas une vitesse de croissance maximum (Raheja, Tepperman et Tepperman, 1975). L'encombrement de la ration et le ralentissement du transit digestif, tendant à diminuer l'ingestion, sont sans doute responsables des baisses de performances. Les résultats contradictoires faisant état d'une semblable aptitude des régimes glucidiques et lipidiques sont fondés sur des essais où les gains de poids ont été plutôt faibles (Renner, 1964).

2. Absorption des hexoses. - Quels que soient le régime et la technique de mesure (in vitro ou in vivo) on observe une vitesse d'absorption beaucoup plus grande pour le glucose que pour le fructose, dans tout l'intestin grêle. La différence entre les deux hexoses correspond sensiblement à celle indiquée dans la littérature (depuis Bogner, 1961). Mais les capacités d'absorption varient d'un segment à l'autre. Dans le jéjunum et l'iléon, l'absorption est plus rapide que dans le duodénum. Chez le poulet comme chez le rat (Pritchard et Porteous, 1977), ce sont donc les parties terminales de l'intestin grêle qui assurent le principal rôle.

Le régime influence la vitesse d'absorption. In vitro, l'accumulation du glucose ef du fructose est supérieure de 30 p. 100 avec le régime gras comparé aux deux régimes glucidiques. Ces derniers assurent des incorporations semblables. In vivo, on obtient des résultats comparables : l'absorption est plus rapide chez les poulets privés de glucides. Bien que la différence avec les deux autres lots soit moins marquée que dans la méthode in vitro, elle demeure en partie significative pour l'absorption du glucose (tabl. 3). Au contraire du rat (Ginsburg et Heggeness, 1968) le poulet ne s'adapte pas en

\section{TABLEAU 3}

Vitesse d'absorption du glucose et du fructose $(\mathrm{mg} / \mathrm{g}$ de poids $\mathrm{sec})$

en fonction du régime alimentaire mesurée in vitro $(20 \mathrm{~min}$. d'incubation) ef in vivo (30 min. de perfusion)

\begin{tabular}{|c|c|c|c|c|c|}
\hline \multirow{2}{*}{\multicolumn{2}{|c|}{ Régime }} & \multicolumn{3}{|c|}{ In vitro } & \multirow{2}{*}{$\frac{\text { In vivo }}{\text { Duodénum }}$} \\
\hline & & \multirow{2}{*}{$\begin{array}{c}\text { Duodénum } \\
\begin{array}{l}3,80 \pm 0,36(A A) \\
3,16 \pm 0,30(a a)\end{array}\end{array}$} & Jéjunum & Iléon & \\
\hline Saccharose & $\begin{array}{l}\text { Glucose } \\
\text { Fructose }\end{array}$ & & $\begin{array}{c}7,20 \pm 1,02(A B) \\
4,10 \pm 0,33(a b)\end{array}$ & $\begin{array}{l}5,54 \pm 0,64\left({ }^{A C}\right) \\
4,06 \pm 0,26(a b)\end{array}$ & $\begin{array}{l}19,90 \pm 1,41\left({ }^{A}\right) \\
15,44 \pm 1,64\left({ }^{a}\right)\end{array}$ \\
\hline Amidon & $\begin{array}{l}\text { Glucose } \\
\text { Fructose }\end{array}$ & $\begin{array}{l}3,58 \pm 0,27(A A) \\
3,32 \pm 0,15(a a)\end{array}$ & $\begin{array}{l}5,27 \pm 0,86\left({ }^{B B}\right) \\
3,84 \pm 0,27(a \alpha)\end{array}$ & $\begin{array}{l}5,65 \pm 0,51\left({ }^{A B}\right) \\
3,96 \pm 0,17(a a)\end{array}$ & $\begin{array}{l}23,33 \pm 2,05\left(^{A B}\right) \\
12,39 \pm 2,07\left({ }^{a}\right)\end{array}$ \\
\hline Graisse & $\begin{array}{l}\text { Glucose } \\
\text { Fructose }\end{array}$ & $\begin{array}{l}5,42 \pm 0,58\left({ }^{B A}\right) \\
4,84 \pm 0,44\left({ }^{b a}\right)\end{array}$ & $\begin{array}{c}10,0 \pm 1,32\left({ }^{C B}\right) \\
5,41 \pm 0,38(b b)\end{array}$ & $\begin{array}{l}9,25 \pm 0,69\left(^{B B}\right) \\
5,67 \pm 0,45\left(^{b b}\right)\end{array}$ & $\begin{array}{l}25,89 \pm 2,48\left({ }^{B}\right) \\
17,79 \pm 1,18(a)\end{array}$ \\
\hline
\end{tabular}

Pour un même segment, la première lettre indique si la différence entre régimes est significative $(P<0,05)$; Pour un même régime, la seconde lettre indique si la différence entre segments est significative $(P<0,05)$. La vitesse d'absorption du glucose est toujours significativement plus élevée que celle du fructose, sauf au niveau du duodénum in vitro $(P<0,05)$. 
absorbant plus rapidement les hexoses apportés par l'alimentation. Le régime sans glucides conduit à l'absorption la plus élevée. Cette stimulation par carence peut être comparée à celle observée à l'état de jeône chez le poulet comme chez le rat (Michael et Hodges, 1973). Elle résulte probablement d'une action directe des aliments sur l'activité métabolique des entérocytes (Levin, 1976).

\section{Conclusion.}

Les performances particulièrement élevées avec le régime riche en saccharose soulignent l'intérêt nutritionnel du sucre pendant la croissance du poulet de chair. Les nutriments énergétiques influencent la physiologie digestive. La vitesse d'absorption intestinale du glucose et du fructose est maximum avec un régime gras. Apparemment, il n'existe pas d'adaptation aux glucides ingérés (saccharose ou amidon). L'absence d'adaptation supporte le concept d'une capacité d'absorption suffisante pour ne pas être un facteur limitant l'utilisation digestive des glucides. Trop rapide, l'absorption des hexoses ne saurait être accélérée par des apports élevés dans l'alimentation du poulet de chair.

\section{Commission CNERNA Digestion-Absorption/Association des Physiologistes, Paris 5-6 octobre 1978.}

\section{Références}

BOGNER, P. H., 1961. Alimentary absorption of reduced sugars by embryos and young chicks. Proc. Soc. exp. Biol. Med., 107, 263-265.

GINSBURG J. M., HEGGENESS F. W., 1968. Adaptation in monosaccharide absorption in infant and adult rats. J. Nufr., 96, 494-498.

MICHAEL E., HODGES R. D., 1973. Histochemical changes in the fowl small intestine associated with enhanced absorption after feed restriction. Hisfochem. J., 36, 39-49.

LEVIN R. J., 1976. Digestion and absorption of carbohydrate from embryo to adult, 63-116. In BOORMAN K. N., FREEMAN B. H., Brit. Poultry Sci. Ltd., Edinburgh.

PRITCHARD P. J., PORTEOUS J. W., 1977. Steady-state metabolism and transport of D-glucose by rat small intestine in vitro. Biochem. J. (G. B.), 164, 1-14.

RAHEJA K. L., TEPPERMAN J., TEPPERMAN H., 1975. Effects of high carbohydrate, high fat or high protein diets on glucose oxydation by isolated intestinal epithelial cells of the chick. J. Nutr., 105, 1433-1439.

RENNER R.. 1964. Factors affecting the utilisation of "Carbohydrate-free " diets by the chick. I. Level of protein. J. Nutr., 84, 322-326.

SIDDONS R. C., 1972. Effect of diet on disaccharidase activity in the chick. Brit. J. Nutr., 27, 343-352. 